\title{
A prospective cohort study to evaluate the correlates of polypharmacy and its association with depression among elderly patients
}

\author{
Shweta Srivatsa, Debdipta Bose*, Sushma M, Jayanthi C.R.
}

Department of Pharmacology, Bangalore Medical College and Research Institute, Bengaluru, Karnataka, India

Received: 30 June 2016 Accepted: 05 August 2016

*Correspondence to:

Dr. Debdipta Bose,

Email: debdipta.bose@gmail.com

Copyright: (C) the author(s), publisher and licensee Medip Academy. This is an open-access article distributed under the terms of the Creative Commons Attribution Non-Commercial License, which permits unrestricted non-commercial use, distribution, and reproduction in any medium, provided the original work is properly cited.

\begin{abstract}
Background: Polypharmacy is a reliable indicator of irrational prescribing particularly among elderly. Polypharmacy increases the risk of adverse drug reactions (ADRs) exponentially imposing higher economic burden. Addressing and evaluating the prescribing practices in elderly will rationalize the drug utilization leading to improvement in quality of health care. The present study was taken to evaluate the determinants of polypharmacy and its association with depression, defined as a 15 item geriatric depression scale (GDS) >6, in elderly patients.
\end{abstract}

Methods: This prospective cohort study was conducted at department of medicine, Victoria hospital, Bengaluru 100 patients aged 60 and above years was enrolled. Relevant data regarding patients' demographic details, smoking and alcohol consumption, medical diagnosis and drug details were collected. Geriatric Depression Scale was used to diagnose depression.

Results: Out of 100 patients screened, 36\% were males and $64 \%$ were females. Polypharmacy was noted in $73 \%$ of the elderly, of which $43 \%$ had cumulative co morbidity ( $\geq 4$ diagnoses). $68 \%$ were found to have a GDS score of $\geq 6$, which corresponded to Depression. Patients with depression (GDS score $\geq 6$ ) had 1.54 (OR-1.54, 95\% CI-0.59-4.01) times more risk of encountering polypharmacy ( $\geq 4$ drugs). Cumulative co-morbidity (OR-1.52, 95\% CI-1.08$2.11, \mathrm{p}<0.05)$ was identified as an independent correlate of polypharmacy.

Conclusions: Increasing age, males, Cumulative comorbidity of $\geq 4$ diagnoses and geriatric depression were found to be the positive correlates of polypharmacy in elderly. Though geriatric depression increases the odds of encountering polypharmacy, it does not affect drug consumption directly; rather it is an important index of cumulative co-morbidity which influences polypharmacy significantly.

Keywords: Elderly, Polypharmacy, Geriatric depression Score, Cumulative co morbidity

\section{INTRODUCTION}

Drugs therapy in elderly is challenging, because of pharmacokinetic changes of ageing which often results in drug-drug interactions leading to disproportionately high rate of ADRs. ADRs are responsible for 3\%-13\% of all the admissions and complicate $5 \%-20 \%$ of hospital stay in patients aged more than 65 years. ${ }^{1}$ Age-related polypathology often demands multiple medications giving rise to polypharmacy among elderly. Polypharmacy increases the risk of drug-related events such as falls, confusion and functional decline in elderly. Polypharmacy, in-turn increases the risks of negative health outcomes like drug interactions, ADRs, hospital admission leading to economic burden. Previous studies have reported depression, cumulative co-morbidity; inappropriate prescribing practice and selected chronic conditions like diabetes mellitus and congestive heart failure as the Positive correlates of polypharmacy. ${ }^{2}$ The other factors negatively influence polypharmacy were identified to be smoking, alcohol consumption, cognitive ability, physical status and ADRs before admission. ${ }^{3}$ Identifying the predictors of polypharmacy in elderly will help to frame interventional strategies to rationalize the prescribing practices.

Psychological well-being is considered as one of the important index of successful aging. Elderly population often succumbs to depression because of multiple medical ailments or individual's self-perceived health. ${ }^{4}$ The association of depression and polypharmacy appears to be bidirectional. Depression was found to be a main 
determinant of drug consumption in Australian homedwelling people aged over $59 .^{5}$ Therefore physicians need to identify the determinants of depression before prescribing for elderly as they are susceptible to develop many complications of drug therapy.

To the best of our knowledge, there are no studies in India analyzing the determinants of polypharmacy among elderly and exploring its association with depression. Hence the present study was undertaken.

\section{METHODS}

\section{Settings and study design}

This cross-sectional study was conducted in the department of Medicine at Bangalore Medical College and Research Institute, Bengaluru.

\section{Study population}

100 consecutively selected patients aged $>60$ years who gave consent voluntarily were enrolled in the study. Patients with diagnosis of depression or anxiety, those who are on antidepressant/anti-anxiety medications and those who failed or unable to complete 15-item GDS scale were excluded from the study.

\section{Data collection}

All relevant data of the patients regarding demographic details (age, gender, smoking and alcohol consumption), clinical characteristics (medical diagnosis, co-morbid illness, number of co-morbidity) and drug details (prescribed drugs, no of drugs) were recorded in predesigned case record forms. Each drug was considered only once during drug data analysis. Explanatory variables like age, gender, smoking and alcohol consumption and cumulative co-morbidity $(\geq 4$ medical diagnosis) were considered to determine the correlates of polypharmacy. The study was commenced after obtaining Institutional Ethics Committee approval. Informed consent was taken from all the participants. The study was conducted in compliance with the Declaration of Helsinki and Indian Good Clinical Practice (GCP) guidelines.

\section{Assessment tools}

Depression was assessed by using 15 items Geriatric Depression Scale, where GDS $\geq 6$ will be considered as depressed. Co-morbid illnesses were diagnosed using International Classification of Disease-10 (ICD-10). ${ }^{6}$

\section{Statistical analysis}

Descriptive statistics like number, percentage were used to analyse the demographical and clinical characteristics. Mean \pm standard deviation was used for the continuous, parametric variables and median and interquartile range for the continuous non parametric variables. Chi-square test or Fisher's exact test was used to determine the association between the demographical, clinical and laboratory variables with the number of drugs $(<4$ drugs and $\geq 4$ drugs) and GDS score $\{<6$ (no depression) and $\geq 6$ (depression) $\}$. Odds ratio (OR) and $95 \%$ confidence interval (CI) of each correlate was analysed to identify the potential predictors of polypharmacy and depression. Multiple logistic regression model was used to analyze the predictors of polypharmacy ( $\geq 4$ drugs prescribed) among elderly patients. After that regression model (adjusted odds ratio [OR]) was adjusted for age and gender to eliminate the effect of confounders. Statistical analysis was done by using Statistical software package STATA/IC version 12.1. $\mathrm{P}<0.05$ is considered as significant.

\section{Ethics}

The study was commenced after obtaining Institutional Ethics Committee approval. Informed consent was taken from all the participants. The study was conducted in compliance with the Declaration of Helsinki and Indian Good Clinical Practice (GCP) guidelines.

\section{RESULTS}

\section{Demographic data analysis}

Among the 100 patients enrolled in the study majority of the subjects were belonged to the age group of 61-70 years $(74 \%)$ with mean age $68.01 \pm 6.26$ years. Our study showed female preponderance $(64 \%)$. Majority of the participants were non-drinkers and non-smokers (56\%) with $22 \%$ being both alcoholic and smokers as shown in Table 1.

Table 1: Depicting the demographic features of the study group $(n=100)$.

\begin{tabular}{|lll|}
\hline Characteristics & Number $(\%)$ & Mean \pm SD \\
\hline Age (years) & & \\
\hline $61-70$ & $74(74.00)$ & $68.01 \pm 6.26$ \\
\hline $71-80$ & $21(21.00)$ & \\
\hline $81-90$ & $5(5.00)$ & \\
\hline Gender & $36(36.00)$ & \\
\hline Male & $64(64.00)$ & \\
\hline Female & $11(11.00)$ \\
\hline Habits & $11(11.00)$ \\
\hline Alcohol & $22(22.00)$ \\
\hline Smoking & $56(56.00)$ \\
\hline Both & \\
\hline No &
\end{tabular}

\section{Clinical characteristics}

On analysis we found that $43 \%$ of the elderly participants had $>4$ cumulative co-morbidity with mean no of co- 
morbidity $3.49 \pm 1.52$ per patients. High no of cumulative co-morbidity lead to higher drug utilization ( $>4$ drugs$73 \%$ ) with mean no drugs $5.29 \pm 2.40$ per patients. Majority $(68 \%)$ of participants had depressive disorders with mean GDS score $6.92+2.44$ as given in Table 2 .

Table 2: Showing clinical characteristics $(n=100)$.

\begin{tabular}{|c|c|c|}
\hline Variables & Number (\%) & Mean \pm SD \\
\hline \multicolumn{3}{|c|}{ No. of drugs } \\
\hline$<4$ & $27(27.00)$ & $5.29 \pm 2.40$ \\
\hline$\geq 4$ & $73(73.00)$ & \\
\hline \multicolumn{3}{|l|}{ GDS score } \\
\hline$<6$ & $32(32.00)$ & $6.92 \pm 2.44$ \\
\hline$\geq 6$ & $68(68.00)$ & \\
\hline \multicolumn{3}{|c|}{$\begin{array}{l}\text { Cumulative co- } \\
\text { morbidity }\end{array}$} \\
\hline$<4$ & $57(57.00)$ & $3.49 \pm 1.52$ \\
\hline$\geq 4$ & $43(43.00)$ & \\
\hline
\end{tabular}

Association of polypharmacy with age, gender and habits

Polypharmacy was noted 1.3 times and 1.8 times more among patients aged between 61-70 years and 81-90 years respectively. Negative association was found with polypharmacy among patients aged between 71-80 years. Occurrence of polypharmacy was 1.17 times higher among elderly males; on the contrary females were negatively associated with polypharmacy. Alcohol and Smoking habits were negatively associated with polypharmacy as presented in Table 3 .

\section{Association of polypharmacy with co-morbidities}

Cumulative -co-morbidity was strongly associated with polypharmacy (OR-3.56, p <0.05). Polypharmacy was observed 2.3 times more among elderly patients with hypertension, 2 times more with diabetes, 3.67 times more with ischemic heart disease, 1.9 times more with

Table 3: Depiction of correlates of polypharmacy.

\begin{tabular}{|c|c|c|c|c|}
\hline \multirow{2}{*}{ Characteristics } & \multicolumn{2}{|c|}{ No. of drugs used (\%) } & \multirow{2}{*}{ OR } & \multirow{2}{*}{$95 \% \mathrm{CI}$} \\
\hline & $<4$ drug users $(n=27)$ & $\geq 4$ drug user $(n=73)$ & & \\
\hline \multicolumn{5}{|l|}{ Age in Years } \\
\hline $61-70$ & $21(77.78)$ & $53(72.60)$ & 1.3 & $0.42-4.5$ \\
\hline $71-80$ & $4(14.81)$ & $17(23.29)$ & 0.57 & $0.12-2$ \\
\hline $81-90$ & $2(7.41)$ & $3(4.11)$ & 1.85 & $0.14-17$ \\
\hline \multicolumn{5}{|l|}{ Gender } \\
\hline Male & $9(33.33)$ & $27(36.99)$ & 1.17 & $0.42-3.2$ \\
\hline Female & $18(66.67)$ & $46(63.01)$ & 0.85 & $0.42-3.2$ \\
\hline \multicolumn{5}{|c|}{ Cumulative co-morbidity } \\
\hline$\geq 4$ & $6(22.22)$ & $37(50.68)$ & 3.56 & $1.2-12$ \\
\hline \multicolumn{5}{|l|}{ Alcohol } \\
\hline Yes & $9(33.33)$ & $24(32.88)$ & 0.97 & $0.35-2.8$ \\
\hline \multicolumn{5}{|l|}{ Smoking } \\
\hline Yes & $18(66.67)$ & $47(64.38)$ & 0.90 & $0.31-2.4$ \\
\hline \multicolumn{5}{|l|}{ Hypertension } \\
\hline Yes & $7(25.9)$ & $33(45.2)$ & 2.3 & $0.82-7.3$ \\
\hline \multicolumn{5}{|l|}{ Diabetes } \\
\hline Yes & $13(48.1)$ & $36(49.3)$ & 2 & $0.84-4.9$ \\
\hline \multicolumn{5}{|c|}{ Ischemic Heart Disease } \\
\hline Yes & $1(3.7)$ & $9(12.3)$ & 3.62 & $0.46-166$ \\
\hline \multicolumn{5}{|l|}{ Dyslipidemia } \\
\hline Yes & $1(3.7)$ & $5(6.8)$ & 1.9 & $0.19-93$ \\
\hline \multicolumn{5}{|c|}{ Chronic Obstructive Pulmonary disease } \\
\hline Yes & $1(3.7)$ & $4(5.5)$ & 1.5 & $0.13-77$ \\
\hline \multicolumn{5}{|l|}{ GDS score $\geq 6$} \\
\hline Yes & $16(59.3)$ & $52(71.2)$ & 1.7 & $0.6-4.7$ \\
\hline
\end{tabular}

dyslipidemia and 1.5 times more with chronic obstructive pulmonary disease. Polypharmacy was noted 1.7 times higher among the elderly patients with depression as presented in Table 3. 
Table 4: Correlates of GDS score among elderly.

\begin{tabular}{|c|c|c|c|c|}
\hline \multirow{2}{*}{ Characteristics } & \multicolumn{2}{|c|}{ GDS Score (\%) } & \multirow{2}{*}{ OR } & \multirow{2}{*}{$95 \% \mathrm{CI}$} \\
\hline & $<6(n=32)$ & $\geq 6(\mathrm{n}=68)$ & & \\
\hline \multicolumn{5}{|l|}{ Age (in years) } \\
\hline $61-70$ & $25(78.13)$ & $49(72.06)$ & 0.72 & $0.22-2.1$ \\
\hline $71-80$ & $5(15.63)$ & $16(23.53)$ & 1.53 & $0.5-6.4$ \\
\hline $81-90$ & $2(6.25)$ & $3(4.41)$ & 0.69 & $0.07-8.7$ \\
\hline \multicolumn{5}{|l|}{ Gender } \\
\hline Male & $13(40.63)$ & $23(33.82)$ & 0.74 & $0.28-1.9$ \\
\hline Female & $19(59.38)$ & $45(66.18)$ & 1.3 & $0.51-3.5$ \\
\hline \multicolumn{5}{|c|}{ Cumulative co-morbidity } \\
\hline$\geq 4$ & $13(40.63)$ & $30(44.12)$ & 1.2 & $0.45-2.9$ \\
\hline \multicolumn{5}{|l|}{ Alcohol } \\
\hline Yes & $7(21.88)$ & $26(38.24)$ & 2.1 & $0.78-6.78$ \\
\hline \multicolumn{5}{|l|}{ Smoking } \\
\hline Yes & $7(21.88)$ & $26(38.24)$ & 2.1 & $0.78-6.78$ \\
\hline \multicolumn{5}{|l|}{ Hypertension } \\
\hline Yes & $16(50)$ & $24(35.2)$ & 0.56 & $0.21-1.3$ \\
\hline \multicolumn{5}{|l|}{ Diabetes } \\
\hline Yes & $19(59)$ & $30(44.1)$ & 0.54 & $0.21-1.3$ \\
\hline \multicolumn{5}{|c|}{ Ischemic Heart Disease } \\
\hline Yes & $2(6.3)$ & $8(11.8)$ & 1.9 & $0.36-20.3$ \\
\hline \multicolumn{5}{|l|}{ Dyslipidemia } \\
\hline Yes & $2(6.3)$ & $4(5.9)$ & 0.93 & $0.12-10.9$ \\
\hline \multicolumn{5}{|c|}{ Chronic Obstructive Pulmonary disease } \\
\hline Yes & $2(6.3)$ & $3(4.4)$ & 0.69 & $0.07-8.7$ \\
\hline \multicolumn{5}{|l|}{ Drug user } \\
\hline$\geq 4$ & $21(65.63)$ & $52(76.47)$ & 1.7 & $0.6-4.7$ \\
\hline
\end{tabular}

Association of GDS scores with age, gender, habits and co-morbidities

Occurrence of depression was 1.53 times higher among the patients aged between 71-80 years. Negative association with depression was noted among patients aged between 61-70 years and 81-90 years. Incidence of depression was 1.3 times higher among females, while male gender was negatively associated with depression. Occurrence of depression was 2.1 times higher among elderly alcoholics and smokers as shown in Table 4.

\section{Association of polypharmacy with co-morbidities}

Depression was 1.2 times higher among elderly with cumulative co-morbidity ( $>4)$. Depression was 1.9 times more common among the elderly patients with ischemic heart disease. Depression was negatively associated with dyslipidemia, chronic obstructive pulmonary disease, hypertension and diabetes as given in Table 4.

\section{Predictors of polypharmacy}

The logistic regression model showed that those patients with GDS score $\geq 6$ have 1.54 times more risk of encountering with polypharmacy. OR and corresponding
95\% CI were calculated after adjusting for age and gender which indicated the similar risk pattern (odds ratio and 95\% CI 1.54 (0.59-4.01). Elderly patients with cumulative co morbidity ( $\geq 4$ diagnoses) had significantly higher chance of utilizing 4 or more no. of drugs (OR1.52 , CI of 1.08-2.11, p <0.05) as given in Table 5.

Table 5: Predictors of polypharmacy.

\begin{tabular}{|lccl|}
\hline Characteristics & OR & $95 \%$ CI & p value \\
\hline $\begin{array}{l}\text { Cumulative } \\
\text { comorbidity }\end{array}$ & $\mathbf{1 . 5 1}$ & $\mathbf{1 . 0 7 - 2 . 1 3}$ & $\mathbf{0 . 0 1 8}$ \\
\hline GDS score $\geq 6$ & $\mathbf{1 . 5 4}$ & $\mathbf{0 . 5 9 - 4 . 0 1}$ & $\mathbf{0 . 3 7 8}$ \\
\hline
\end{tabular}

$\mathrm{P}<0.05$ considered as significant

\section{DISCUSSION}

The purpose of this study was to determine the correlates of polypharmacy in elderly patients with special emphasis on association of polypharmacy with depression.

Among the elderly patients enrolled in the study, 74\% were between 61 to 70 years with Female preponderance (64\%). A study by Ayesha $\mathrm{R}$ from the same centre in 2010, noted $66 \%$ from 60-69 years age group and male 
preponderance. $^{7}$ This result may indicate improving health awareness among female elderly population. Another study from Taiwan also indicates female preponderance of $61 \%{ }^{8}$

Polypharmacy was noted among $73 \%$ with the average number of $5.29 \pm 2.4$ drugs per prescription. A study by $\mathrm{Al}$ Ameri et al in UAE reported that $89 \%$ of the study population received more than five drugs and increasing age and comorbidities were the main correlates of polypharmacy. ${ }^{9}$ Ayesha et al noted $8.42 \pm 2.4$ drugs per prescription in the same center. ${ }^{7}$ The reduction in the average number of drugs per prescription indicates efforts towards optimization of therapy among elderly in our center. As polypharmacy increases the risk of adverse drug reactions and potential drug-drug interactions in elderly, efforts should be made to rationalize prescribing practices.

Cumulative co morbidities $\geq 4$ was noted among $43 \%$ of the patients. The most common co-morbidities associated were hypertension, diabetes mellitus, COPD, Ischemic heart disease, thyroid disorders, epilepsy, upper GI distress, chronic constipation and skin disorders. This reflects the general affliction pattern in Indian elderly patients with cardiovascular disorders taking the lead. Al Ameri et al from UAE reported a significant association of hypertension, dyslipidemia and diabetes with polypharmacy in elderly. ${ }^{9}$

GDS score of $\geq 6$ indicating depression was seen among $68 \%$ of the patients. Pilania et al from India in 2013 reported overall prevalence of geriatric depression as $22 \%{ }^{10}$ Rising prevalence of chronic non-communicable diseases, societal modernization and marginalization of elderly population may impose detrimental impact on the psychological health of the elderly leading to higher incidence of depression among them.

In this study, we evaluated the variables associated with polypharmacy in elderly as in Table 3 . The positive correlates were found to be higher age group of 81-90 years, males, cumulative comorbidities $\geq 4$, mainly hypertension, diabetes, IHD, dyslipidemia and COPD. GDS scores of $\geq 6$ indicating depression was also found to be positively associated with polypharmacy. Study of global ageing and adult health (SAGE) conducted in association with WHO in India in 2007, revealed a prevalence of $4.2 \%$ of polypharmacy among elderly in India. Increasing age, males, self-reported bad health, diabetes, depression and hypertension were noted to be the main predictors of polypharmacy in SAGE. ${ }^{11}$ Hajjar et al conducted a review of literature (1986 to 2007 MEDLINE database) on polypharmacy in elderly in 2007 and reported that increased age, white race, education, poorer health, depression, hypertension, diabetes, asthma, anaemia, angina, osteoarthritis, gout, diverticulosis were mainly associated with polypharmacy. ${ }^{12}$

A systematic review by Achala et al reported that $33 \%$ of the urban and $25 \%$ of the rural Indians are hypertensives. ${ }^{13}$ The study also states that India harbours $17.8 \%$ of the hypertensives in the world. Veena et al from Bengaluru noted anti hypertensives as the second highest group (10\%) to contribute for polypharmacy in elderly. ${ }^{14}$ Fadare et al from Nigeria reported $30.6 \%$ of the prescriptions were anti hypertensives in elderly. ${ }^{15} \mathrm{We}$ noted that Hypertension increases the risk of polypharmacy by 2.3 times.

Diabetes which is a potential epidemic in India harbours 62 million diabetics presently, which is projected to increase to 72.4 million by $2030 .{ }^{16}$ In the present study, we noted that elderly with diabetes had twice the risk of encountering polypharmacy. A review by Good et al states that need for tight glycemic control and coexisiting conditions like hypertension, dyslipidemia, coronary artery disease and neuropathy mainly contribute towards polypharmacy among elderly diabetics. ${ }^{17}$

In the present study, patients with IHD had 3 times more risk of encountering polypharmacy. Vyas et al from USA estimated the rate of polypharmacy in three chronic condition cluster and reported that individuals with cardiometabolic disease cluster (hypertension, diabetes, heart diseases) only had higher association with polypharmacy as compared to musculoskeletal cluster only (osteoporosis, arthritis) and respiratory cluster only (COPD, asthma). ${ }^{18}$

The negative correlates of polypharmacy were alcohol and smoking habits. The same was reported by Antonelli et al from Italy in a study to evaluate the correlates of polypharmacy in elderly. ${ }^{2}$ Under-reporting of the medical problems and under-treatment of medical ailments commonly seen among patients with smoking and alcohol addiction could be responsible for their negative association with polypharmacy. Geriatric depression has been noted as a correlate of polypharmacy in elderly, as it negatively affects the perception of the disease status. Hence, we evaluated the variables associated with depression among elderly as in Table 4. Increase in age, polypharmacy, cumulative comorbidities $\geq 4$, mainly ischemic heart disease, smoking and alcohol habits increased the odds of encountering depression among elderly. A study conducted by Anwar et al in Karachi reported a prevalence of geriatric depression as $40.6 \%$ with a higher preponderance in women which matches with our results (66\% vs $34 \%$ females). ${ }^{19}$

Smoking was found to be associated with GDS score $>6$ (38\% vs $21 \%$ ) in the present study as reported by Stafford et al in Melbourne, Australia. The reciprocal relationship between smoking and depression could be because of its addictive action via the dopaminergic reward pathway that plays a key role in depression. Smoking is known to dysregulate the striatal $D_{2}$ receptor leading to depressed mood. ${ }^{20}$

In the present study GDS score of $\geq 6$ was commonly observed among those with $\geq 4$ diagnoses ( $41 \%$ vs $44 \%$ ). Antonelli et al from Italy also noted a strongly 
association with co-morbidity $(32.7 \%$ patients with $\geq 4$ diagnoses vs $24.7 \%$ patients with $\leq 4$ diagnoses, $p$ $<0.001)$. A study conducted by Wong et al in Hong-Kong also found increased odds of having clinically relevant depressive symptoms as the number of chronic medical condition increases (OR-1.27; 95\% CI-1.16-1.39). ${ }^{2,21}$ Multiple co-morbidities at older age can impose a negative impact in their mental health leading to depressive symptoms. A retrospective review of $>875,000$ patients in long-term care by Macready et al from American Geriatric Society also inferred polypharmacy as a determinant of depression in elderly patient. The review showed that risk of depression was significantly increased when patients were taking at least nine medications (OR-1.9). ${ }^{22}$ This suggests polypharmacy might negatively affect the perception of disease status leading to depression.

Limitations in our study were inevitable as it was done as a cross sectional analysis. We could not assess the severity of co morbid illnesses, which also contributes to the pathogenesis of depression in elderly. Larger sample size and prospective design would have been better to generalize the results.

\section{CONCLUSION}

We noted polypharmacy in $73 \%$ of the elderly patients enrolled in the present study. A significant association of polypharmacy was noted with cumulative comorbidities especially hypertension, diabetes and IHD. $68 \%$ of the elderly had GDS score $\geq 6$ and was one of the factors linked with polypharmacy. Strategies to optimize the drug therapy in elderly can be designed based on the determinants identified in the present study.

\section{ACKNOWLEDGEMENTS}

The authors would like to thank the faculty, post graduate students and HOD of the department of medicine for their cooperation and support in conducting the project work.

\section{Funding: No funding sources}

Conflict of interest: None declared

Ethical approval: The study was approved by the Institutional Ethics Committee

\section{REFERENCES}

1. Somers A, Petrovic M, Robays H. Reporting adverse drug reactions on a geriatric ward: a pilot project. Eur J Clin Pharmacol. 2003;58:707-14.

2. Antonelli Incalzi R, Corsonello A, Pedone C. Depression and drug utilization in an older population. Ther Clin Risk Manag. 2005;1:55-60.

3. Corsonello A, Pedone C, Corica F, Antonelli Incalzi R. Polypharmacy in elderly patients at discharge from the acute care hospital. Ther Clin Risk Manag. 2007;3(1):197-203.
4. Mulsant BH, Ganguli M, Seaberg. Thc rc!aticnshipbetween selt $\sim$ rated health and depressive symptoms in an epidemiological sample of communitydwelling older adults. Journal of the American Geriatric Society. 1997;45(8):954-8.

5. Simons LA, Tett S, Simons J. Multiple medication use in the elderly. Use of prescription and nonprescription drugs in an Australian community setting. Med J Aust .1992;157:242-6.

6. Lesher EL, Berryhill JS. Validation of the Geriatric Depression Scale-Short Form among inpatients. J Clin Psychol. 1994;50:256-60.

7. Ayesha R, Laxminarayana K, Astha Sarda, Sushma M, Jayanthi CR. Polypharmacy leading to adverse drug reactions in elderly in a tertiary care hospital. Int J Pharm Bio Sci. 2012;3(3):218-24.

8. Chan DC, Hao YT, Wu SC. Characteristics of outpatient prescriptions for frail Taiwanese elders with long-term care needs. Pharm Drug Saf. 2009;18(4):327-34.

9. Al Ameri MN, Makramalla E, Albur U, Kumar A, Rao P. Prevalence of Poly-pharmacy in the Elderly: Implications Page 4 of 7 of Age, Gender, Comorbidities and Drug Interactions. SOJ Pharm Pharm Sci. 2014;1(3):1-7.

10. Pilania M, Bairwa M, Kumar N, Khanna P, Khurana H. Elderly depression in India: An emerging public health challenge. AMJ. 2013;6(3):107-11.

11. Mili D. Prevalence and risk factors of polypharmacy among elderly in India: Evidence from SAGE Data. International Journal of Public Mental Health \& Neurosciences. 2015;2(2):11-16.

12. Hajjar ER, Cafiero AC, Hanlon JT. Polypharmacy in elderly patients. Am J Geriatr Pharmacother. 2007;5:345-51.

13. Raghupathy A, Nanda KK, Hira P, Hassan K, Oscar FH, Di Emanuele A, et al. Hypertension in India: a systemic review and meta-analysis of prevalence, awareness, and control of hypertension. Journal of Hypertension. 2014;32(6):1170-7.

14. Veena DR, Padma L, Sapna P. Drug prescribing pattern in elderly patients in a teaching hospital. Journal of Dental and Medical Sciences. 2012;1(5):39-42.

15. Joseph FO, Segun AM, Olumide OA, Rachel AA. Prescription pattern and prevalence of potentially inappropriate medications among elderly patients in a Nigerian rural tertiary hospital. Ther Clin Risk Manag. 2013;9:115-20.

16. Kaveeshwar SA, Cornwall J. The current state of diabetes mellitus in India. AMJ. 2014;7(1):45-8.

17. Chester GB. Polypharmacy in elderly patients with diabetes. Diabetes Spectrum. 2002;15(4):240-8.

18. Vyas A, Pan X, Sambamoorthi U. Chronic Condition Clusters and Polypharmacy among Adults. Int J Fam Med. [Internet]. 2012 Available from: http://www.ncbi.nlm.nih.gov/pmc/articles/ PMC3415173/. Accessed on June 2016. 
19. Bhamani MA, Karim MS, Khan MM. Depression in elderly in Karachi, Pakistan: a cross sectional study. BMC Psychiatry. 2013;13:18.

20. Stafford L, Berk M, Jackson HJ. Tobacco smoking predicts depression and poorer quality of life in heart disease. BMC Cardiovasc Disord. 2013;13:210 .

21. Wong SYS, Mercer SW, Jean W, Jason L. The influence of multi morbidity and self-reported socio economic standing on the prevalence of depression in an elderly Hong Kong population. BMC Public health. 2008;8:119.
22. Macready N. Los Angeles Bureau. Polypharmacy May Be Linked to Depression in the Elderly. www.eclinicalpsychiatrynews.com. Accessed on Jan 2005 .

Cite this article as: Srivatsa $S$, Bose D, Sushma M, Jayanthi CR. A prospective cohort study to evaluate the correlates of polypharmacy and its association with depression among elderly patients. Int J Basic Clin Pharmacol 2016;5:1984-90. 